\title{
Music and emotion: perceptual determinants, immediacy, and isolation after brain damage
}

\author{
Isabelle Peretz*, Lise Gagnon, Bernard Bouchard \\ Department of Psychology, University of Montreal, and Research Center \\ of the University Hospital of Geriatry of Montreal, C.P. 6128, \\ succ. Centre-ville, Montréal, Québec H3C 3J7, Canada
}

Received 19 December 1997; accepted 5 June 1998

\begin{abstract}
This study grew out of the observation of a remarkable sparing of emotional responses to music in the context of severe deficits in music processing after brain damage in a nonmusician. Six experiments were designed to explore the perceptual basis of emotional judgments in music. In each experiment, the same set of 32 excerpts taken from the classical repertoire and intended to convey a happy or sad tone were presented under various transformations and with different task demands. In Expts. 1 to 3, subjects were required to judge on a 10-point scale whether the excerpts were happy or sad. Altogether the results show that emotional judgments are (a) highly consistent across subjects and resistant to brain damage; (b) determined by musical structure (mode and tempo); and (c) immediate. Experiments 4 to 6 were designed to assess whether emotional and non-emotional judgments reflect the operations of a single perceptual analysis system. To this aim, we searched for evidence of dissociation in our brain-damaged patient, I.R., by using tasks that do not require emotional interpretation. These non-emotional tasks were a 'same-different' classification task (Expt. 4), error detection tasks (Expt. 5A,B) and a change monitoring task (Expt. 6). I.R. was impaired in these non-emotional tasks except when the change affected the mode and the tempo of the excerpt, in which case I.R. performed close to normal. The results are discussed in relation to the possibility that emotional and non-emotional judgments are the products of distinct pathways. (C) 1998 Elsevier Science B.V. All rights reserved
\end{abstract}

Keywords: Music; Emotion; Perception; Amusia; Brain; Mode; Tempo

* Corresponding author. Fax: +1 514 3435787; e- mail: peretzi@magellan.umontreal.ca 


\section{Introduction}

Music is often characterized as the language of emotions. Ironically, the vast majority of experimental studies has been devoted to the study of musical structure as a non-verbal language, rarely as an emotional language. Part of this situation can be attributed to the widely held belief that emotional interpretation of music is a highly personal and variable experience, hence escaping scientific examination. A fortiori, emotional interpretation of music is not conceived as the product of a neuro-anatomical arrangement that can be shared by most members of a given musical culture. The present study shows that this view is misleading.

We present the case of a woman, I.R., who suffers from a severe loss of music recognition and expressive abilities, and yet retains the capacity to appreciate music. I.R. is a right-handed 40-year-old woman with 10 years of formal education. She sustained, 10 years prior to the present study, successive brain surgeries for the clipping of mirror aneurysms on each middle cerebral artery. As a result, sequelar lesions were found in both temporal lobes, extending bilaterally to frontal areas. At the time of testing, I.R.'s intellectual and memory abilities corresponded to a normal level for her level of education and age. Speech comprehension and expression were also normal except for a mild articulatory deficit. Contrasting with normal level of mental functioning, I.R. experiences persistent difficulties with music. She can no longer recognize melodies that were once highly familiar to her; she is unable to discriminate musical sequences. She can no longer sing more than a single pitch, even though she used to sing before her brain accident. Therefore, her musical abilities were found to be severely impaired while her speech and intellectual functions were spared. This pattern corresponds to a clear-cut case of amusia without aphasia in neuropsychological terms (Peretz et al., 1997).

Despite I.R.'s clear deficit in music, of which she is fully aware, she claims that she still enjoys music. This is a non-trivial claim. How can someone derive a proper emotional interpretation of music without an adequate structural analysis of it? This is the question experimentally addressed in the present study. To our knowledge, no prior study has assessed emotional treatment of music in brain-damaged populations. Consequently, no dissociation between emotional and structural judgments has ever been envisaged. The present study constitutes a first exploration in this vast domain of music emotion and cognition.

First, we will present the neuropsychological background for the present study. Then, we will present each experimental investigation in the order in which the patient and her matched control subjects were evaluated. The rationale for following the same experimental paths (except for Expt. 5B which took place a year after completion of the study) are that (1) the same musical stimuli were used across experiments, thus becoming progressively more familiar to the subjects as we proceed, and (2) the readers will get a share of the excitement and bewilderment that we experienced while conducting the present study. 


\section{Case summary}

\subsection{Neurology}

I.R. is a right-handed woman in her early forties who suffered bilateral cerebral damage caused by the repair of cerebral aneurysms located on the left and right middle cerebral arteries. At the time of testing, 10 years after the brain surgeries, CT scans showed that in the left hemisphere, most of the superior temporal gyrus was damaged; only a small portion of the posterior superior temporal gyrus was spared. The hypodensity extended anteriorly into the frontal operculum, medially into most of the insula, inferiorly into the middle and inferior temporal gyri and posteriorly into the anterior parietal lobule. In the right hemisphere, approximately the anterior one-third of the superior temporal gyrus was infarcted. There was also extensive infarction of the right inferior and middle frontal gyri. Images of the scans as well as more detailed neurological information can be found in Patel et al. (1998).

At the time of testing, I.R. readily engages in conversation with a definite sense of humor. Her only obvious problems are a mild articulation difficulty and a left hemiplegic arm. She also complains of persisting difficulties with music. She can no longer recognize familiar melodies and can no longer sing. I.R. received little formal musical education but was raised in a musical environment since her grandmother and her brother are both professional musicians. I.R. had 10 years of school education and worked as a restaurant manager before her brain accident.

\subsection{Neuropsychology}

A summary of I.R.' s cognitive and auditory functioning is presented in Table 1. I.R.'s latest assessment with the WAIS-R indicates a normal intelligence for her education and a normal memory functioning, with the exception of an impaired short-term memory for digits. There is no evidence of impairment of basic auditory functions, with a normal audiometry. She also exhibits normal scores on language comprehension and discrimination tests (see Peretz et al., 1997 for more details, and Patel et al., 1998 for an examination of prosodic aspects). She has no difficulty recognizing familiar environmental sounds as long as these do not involve musical instruments or musical excerpts. However, on the latter, I.R. is severely impaired.

She cannot name a single melody among those which were highly familiar to her prior to her brain accident. She does show improvement when multiple choices are provided to her without, however, reaching normal performance. I.R. presents thus auditory agnosia which is most prominent for musical events (see Peretz et al., 1997 for more details).

As can be seen in Table 1, when the tests are not standardized, we used the scores provided by four normal subjects for comparison. These control subjects were righthanded female non-musicians whose ages and socio-economic backgrounds closely match with those of I.R. (mean age: 41.2 years, range: $33-48$ years; mean education: 12.7 years, range: $11-14$ years); These control subjects will provide the comparison data in all subsequent investigations as well. 
Table 1

I.R.'s performance on tests of intelligence, memory, audition (language and music) and averaged percentages of correct responses of four matched controls for non-standardized tests, with their lowest and highest scores in parentheses

\begin{tabular}{|c|c|c|c|}
\hline & & I.R. & Matched controls \\
\hline \multicolumn{4}{|c|}{ Intelligence } \\
\hline WAIS-R & Full scale I.Q. & 94 & - \\
\hline \multicolumn{4}{|l|}{ Memory } \\
\hline WAIS-R & Full scale M.Q. & 99 & - \\
\hline \multicolumn{4}{|l|}{ Audition } \\
\hline & Audiometry & Normal & \\
\hline \multicolumn{4}{|c|}{ Language } \\
\hline & Token test & $56 / 62$ & - \\
\hline & Auditory lexical decision & $77 / 80$ & - \\
\hline & Word discrimination & $36 / 36$ & - \\
\hline \multicolumn{4}{|c|}{ Environmental sounds } \\
\hline & Naming response & $40 / 45(88.9 \%)$ & $82.0 \%(75.0-88.9)$ \\
\hline \multicolumn{4}{|c|}{ Familiar music } \\
\hline & Naming response & $0 / 77$ & \\
\hline & Forced-choice (4 titles) & $34 / 77(44.2 \%)$ & $88.8 \%(84.7-93.6)$ \\
\hline \multicolumn{4}{|c|}{ Unfamiliar music } \\
\hline & Discrimination $^{\mathrm{a}}$ & & \\
\hline & Pitch variations & $45 / 90(50.0 \%)$ & $88.1 \%(76.7-96.7)$ \\
\hline & Temporal variations & $15 / 30(50.0 \%)$ & $96.7 \%(93.3-100)$ \\
\hline & Recognition memory $^{\mathrm{a}}$ & $15 / 30(50.0 \%)$ & $86.7 \%(76.7-96.7)$ \\
\hline & Scale-error detection $^{\mathrm{a}}$ & $17 / 30(56.7 \%)$ & $72.7 \%(63.3-86.7)$ \\
\hline & Tonal closure ${ }^{\mathrm{a}}$ & $21 / 32(65.6 \%)$ & $91.0 \%(83.3-96.7)$ \\
\hline
\end{tabular}

${ }^{\mathrm{a}}$ Chance level is $50 \%$.

As a departure point for the present study, I.R.'s ability to process music was tested with a battery of subtests, henceforth referred to as screening tests. Full description of the original tests is found in Peretz (1990) and Liégeois-Chauvel et al. (1998). These screening tests, in which the material is kept as natural and constant across conditions as possible, aim to assess the various components that are known to contribute to music processing. The stimuli were novel musical sequences, played one note at a time on a piano, and were written in accordance to the rules of the tonal structure of the Western idiom. The sequences were 4 bars long, lasted about $4 \mathrm{~s}$ and contained from 8 to 19 tones (mean: 10.7). These melodies were arranged in various tests so as to assess abilities to discriminate pitch and rhythmic variations, to recognize musical sequences heard in prior tests of the battery, and finally to use tonal knowledge in on-line perceptual judgments.

As can be seen in Table 1, I.R. performs at chance in the discrimination of pitch and temporal deviations in a 'same-different' classification task. She is unable to employ melodic cues such as a melodic contour change or a scale violation to discriminate the melodies. Similarly, she is unable to use temporal grouping in rhythmic discrimination tests. When tested for memory recognition of these melodies, I.R. also fails to discriminate them from distractors. I.R. also performs below her controls when presented with the same melodies in on-line tasks. When required 
to either detect a pitch change which violates the scale structure in half the melodies (see scale-error detection test in Table 1) or to judge the adequacy of the final pitch as an ending (which is either the original tonic pitch or a non-triad diatonic tone; this is referred to as tonal closure in Table 1), I.R. exhibits again poor performance. The severity of these musical deficits has been replicated later on with a series of tests using familiar melodies (Peretz and Gagnon, 1998). In sum, I.R. does not give the slightest indication that she can perceive and/or interpret pitch and temporal variations in melodies. This explains why we were intrigued by I.R.'s claim that she still enjoys music.

\section{Experimental investigations}

\subsection{General method}

\subsubsection{Material and apparatus}

The same set of 32 musical selections (presented in Appendix A) was used throughout the experiments. All excerpts were instrumental in that they were not originally sung with lyrics; they were drawn from the corpus of Western music and were thus composed for other purposes than experimentation. The excerpts were chosen from the classic literature because it was considered important to use musical material that is sufficiently complex and engaging to guarantee its processing as a meaningful structure rather than as a simple sequence of tones. The selections were drawn from baroque (e.g. Bach, Albinoni), classical (e.g. Mozart), romantic (e.g. Verdi), and contemporary (e.g. Ravel) periods. These excerpts were selected so that half evoked a sense of happiness and the other half, a sense of sadness. The original instrumentation was generally orchestral (22 excerpts) or for piano solo (eight excerpts). The selections lasted from 7 to $33 \mathrm{~s}$ (mean: $15.8 \mathrm{~s}$ ). The computer-generated versions were created on a microcomputer running a MIDI sequencing program (Sequencer Plus Gold) and controlling a sample playback digital synthesizer (Rolland Sound Canevas SC 50) by entering manually the original scores. Each tone occupied its precise value in terms of pitch and duration, keeping intensity and velocity constant. The pedal was used in the piano renditions.

\subsubsection{Procedure}

All stimuli were recorded onto digital tapes and presented via a Tascam DA-30 digital recorder in free field at a loudness level that was comfortable and customary for the listener. Each subject was tested individually. The control subjects were the same as those used in the screening tests. All subjects were regularly tested over two years.

\subsection{Experiment 1: emotional judgments}

The first step was to objectively evaluate whether or not I.R. was capable of judging the emotional character of the music presented. To this aim, I.R. as well 
as the matched controls were presented with the selections of pre-existing instrumental music which had to be judged on a 10-point scale, with 1 meaning 'sad' and 10, 'happy'. Three versions of the same set of 32 excerpts were used. One version was 'natural' in that the excerpts were directly taken from commercial recordings. The second version had similar instrumentation but was devoid of human expression since it was computer-generated without nuances and with mechanical precision. The third version was also computer-generated but was transcribed into a piano version (the eight excerpts which were originally written for piano remained as such). The three versions were presented in that order in three separate sessions. The goal was to assess the contribution of human expressiveness and of instrumentation to the emotional evaluation of the excerpts.

\subsubsection{Results and discussion}

The mean ratings obtained for the 'happy' and 'sad' selections provided by I.R. and her controls are presented in Table 2 . Throughout the paper, we will consider the excerpts as happy or sad according to the a priori classification (and not according to subjects' judgments). The ratings obtained for each selection by I.R. and averaged across controls were submitted to separate ANOVAs, both considering items as the random variable, Version (natural vs. synthesized vs. piano) as the within-items variable, and Category (happy vs. sad) as the between-items variable. All subjects exhibited a clear distinction between the happy and sad selections, with a main response Category effect of $F(1,30)=310.27$ and 350.84, both $P<0.001$, for I.R. and controls, respectively. The version presented had some influence on ratings (the interaction between response Category and Version reached significance for normals, with $F(2,60)=4.47, P<0.02$, as well as for I.R., with $F(2,60)=8.17$, $P<0.001)$. To specify the source of this difference, additional comparisons between the natural version and each computer-generated version were performed by way of separate ANOVAs. The only comparison to reach statistical significance was found between the natural and the synthesized version in the form of an interaction between Version and response Category, with $F(1,30)=8.82$ and 4.74 , $P<0.05$, for controls and I.R., respectively. As can be seen in Table 2, the synthesized version was found to elicit less extreme judgments than both the natural and piano versions for I.R. as well as her controls.

When scores below 5 and above 6 were dichotomized so as to obtain a percentage of correct classifications as 'happy' and 'sad' music, I.R. obtained 97\%, 88\%, $97 \%$

Table 2

Mean ratings for I.R. and her four matched controls on the three versions of the musical excerpts presented in Expt. 1

\begin{tabular}{|c|c|c|c|c|}
\hline \multirow[t]{2}{*}{ Version } & \multicolumn{2}{|l|}{ I.R. } & \multicolumn{2}{|c|}{ Matched controls } \\
\hline & Happy & $\mathrm{Sad}$ & Happy & $\mathrm{Sad}$ \\
\hline Natural & 9.0 & 3.3 & 8.9 & 3.3 \\
\hline Synthesized & 8.3 & 4.3 & 8.3 & 3.1 \\
\hline Piano-transcription & 9.1 & 2.2 & 8.7 & 3.6 \\
\hline
\end{tabular}


correct and her controls 94\%, 93\%, 95\%, for the natural, synthesized and piano version, respectively. Thus, consensus with the a priori classification into happy and sad music was high across the versions presented. These results indicate that (a) emotional interpretation can reach a high level of consensus, and is, hence, amenable to scientific study, and (b) I.R. displays normal functioning at that level.

None of the excerpts sounded familiar to I.R. When prompted with the titles of the excerpts instead of the music, I.R. claimed that she was familiar with at least four of them (the two Verdi excerpts, Vivaldi and Aranguez). Control subjects judged on average 14 out of the 32 excerpts to be familiar prior to this study. Consequently, the material sounded more familiar to controls than to I.R.

Despite this difference in familiarity with the material, all subjects, including I.R., performed the emotional classification with ease, be the musical selections performed by humans or by a computer. The synthesized version appears, however, to be slightly less well categorized than the piano version. It should be recalled that the piano version was also computer-generated. One likely source for the depressed performance observed with the synthesized orchestral version is that some of the digitized instruments (e.g. the string instruments) sounded artificial. However, this possible interfering effect was small. More generally, it seems that the happy-sad distinction is not conveyed much by human interpretation but is rather determined by the structure of the pieces selected.

\subsection{Experiment 2: structural determinants of emotional judgments}

Since instrumentation and interpretation are not major determinants of emotional judgments in the present study, the excerpts must contain structural characteristics that are relevant in this respect. Two structural properties that are known to convey important information with regard to the happy-sad distinction are the tempo and the mode of the piece (e.g. Hevner, 1935, 1937). Slow tempi, or few beats per minute, tend to evoke sad moods whereas fast tempi, or many beats per minute, tend to evoke happy moods. Mode is related to the subset of pitches selected in a given musical segment: the minor mode is associated with a sad tone whereas the major mode is associated with a happy tone (see Crowder, 1984 for a review).

The musical selections used in the present study generally followed these principles: the happy selections were mostly played in a fast tempo (the quarter note value varies from 80 to 255, conventionally written by M.M. for Metronome Marking ${ }^{1}$ and were all written in a major mode; most sad selections were played at a slow tempo (between 20 and 100 M.M.) and written in the minor mode (see Appendix A). Therefore, it is likely that both tempo and mode are structural characteristics of the pieces that contribute to the happy-sad discrimination.

In order to assess the respective contribution of tempo and mode to the emotional evaluation of the musical stimuli, we manipulated these characteristics orthogonally, in three different conditions. In the tempo condition, all tempi were set to an

\footnotetext{
${ }^{1}$ The values refer here to the notated convention. The values should not, however, be confounded with the perceived beat which, depending on tempo, can occur at a slower rate, corresponding to an integer subdivision of the value given in Appendix A.
} 
Table 3

Mean ratings for I.R. and her four matched controls in the different conditions defined by the type of modification applied to the musical excerpts in Expt. 2

\begin{tabular}{|c|c|c|c|c|}
\hline \multirow[t]{2}{*}{ Condition } & \multicolumn{2}{|l|}{ I.R. } & \multicolumn{2}{|c|}{ Matched controls } \\
\hline & Happy & Sad & Happy & Sad \\
\hline Original & 9.1 & 2.2 & 8.7 & 3.6 \\
\hline Tempo change & 6.8 & 3.4 & 7.5 & 4.2 \\
\hline Mode change & 7.5 & 3.4 & 7.9 & 4.2 \\
\hline Tempo + mode change & 5.8 & 3.9 & 6.6 & 5.0 \\
\hline
\end{tabular}

unique value $^{2}$ (with the quarter note $=84$ M.M.) that corresponds to the median of the original tempi. In the mode condition, the pieces were transcribed in the opposite mode with respect to its original mode. The procedure used for the mode transcription was conventional and identical to the one used by Hevner (1935). Finally, to examine the joint influence of mode and tempo, the two modifications applied in isolation in the mode and tempo conditions were combined in the mode + tempo condition.

The task of the subjects was the same as in the previous experiment. They were requested to judge on a 10-point scale whether each excerpt sounded happy or sad. They were presented with the tempo condition, the mode condition and the mode + tempo condition in that order, in separate sessions. Subjects were not informed of the nature of the changes applied to the stimuli. The version used throughout the three conditions, as well as in all following experiments, was the piano-synthesized version because both I.R. and her controls performed well on that version. Such a version was also easier to manipulate by way of computer-programming. The piano version used in Expt. 1 served here as the control condition which is referred to as the original condition.

\subsubsection{Results and discussion}

The mean ratings obtained by I.R. and her controls in each condition for the 'happy' and 'sad' selections are presented in Table 3. The ratings obtained for each selection by I.R. and averaged across controls were submitted to separate ANOVAs, both considering items as the random variable, Condition (original vs. tempo vs. mode vs. tempo + mode) as the within-items variable and response Category (happy vs. sad) as between-items variable. The analyses reveal that both I.R. and her controls are sensitive to the structural manipulations. Their ratings are significantly influenced by the condition presented; the response Category by Condition interaction is significant in both I.R.'s and the controls' data with $F(3,90)=15.83$ and 24.31, $P<0.001$, respectively. Comparisons between conditions were performed two by two, by separate ANOVAs, considering the same factors as in the overall ANOVA. These subsequent analyses indicate that each

\footnotetext{
${ }^{2}$ In the mode condition, the excerpts were transcribed to the opposite mode. The equivalent transformation for tempo would be to make the fast excerpts slow and the slow excerpts fast. Such tempo inversions are, however, too drastic; the resulting excerpts mostly sounded unnatural or ill-formed. Therefore, we decided to 'neutralize' the tempo instead, by selecting a unique median value.
} 
modification applied to the original version significantly affects the response pattern. When compared with the original version, the tempo condition yields a significant interaction between Condition and response Category (with $F(1,30)=21.38$ and 32.76, $P<0.001$, for I.R. and controls, respectively), as does the mode condition (with $F(1,30)=13.54$ and 13.63, $P<0.001$, for I.R. and controls, respectively) and the tempo + mode condition (with $F(1,30)=46.88$ and 59.66, $P<0.001$, for I.R. and controls, respectively). Note that in the latter condition, where the manipulation has the greatest impact, ratings are still significantly higher for the happy than sad excerpts $\left(t_{30}=2.838\right.$ and 3.718 , both $P<0.01$ by bilateral tests, for I.R. and controls, respectively). Thus, despite the change of both mode and tempo applied to the stimuli, subjects were still able to discriminate happy from sad excerpts. This suggests that other determinants than tempo and mode play a role in the emotional classification of the stimuli ${ }^{3}$.

In summary, both mode and tempo modifications impair performance, particularly when the presented musical excerpts incorporates both modifications. The results support the widely-held notion that both mode and tempo convey important information with regard to the happy-sad classification. Moreover, I.R.'s responses are strikingly similar to those of normal subjects, hence showing spared ability to employ tempo and mode as cues for emotional interpretation. The results show that (a) emotional appraisal is to a large extent determined by musical structure, and (b) I.R.'s emotional judgments reveal normal structural analysis of the musical input, hence leading to opposite conclusions to the ones derived from the previous nonemotional tests (see Table 1).

\subsection{Experiment 3: length effect on emotional judgments}

In Expts. 1 and 2, we showed that I.R. was able to pick up normally abstract pitch information, such as that defining mode, when required to make emotional judgments about the stimuli. In contrast, we know from prior work (Peretz et al., 1997; see Table 1 for a summary) that I.R. is poor at detecting obvious changes on the pitch dimension when required to perform non-emotional tasks such as melody discrimination, scale error detection or closure judgments. One possible account for this discrepancy between emotional and non-emotional judgments may be related to a simple difference in information redundancy in the stimuli. Emotional judgments are fed by recurrent relevant information where both mode and tempo characteristics can be derived from almost every single musical event contained in a well-formed melody with accompaniment. In contrast, error detection or melody discrimination require consideration of only a limited number of events that bear the critical errors or changes. This lack of redundancy in non-emotional judgments may be more

\footnotetext{
${ }^{3}$ The contribution of other structural characteristics than tempo and mode has been explored. The role of harmony was studied through presentation of the melodic line of the excerpts without accompaniments. I.R.'s ratings were 2.9 and 7.8 for the sad and happy excerpts, respectively, and controls' ratings were 3.0 and 8.4 for the sad and happy excerpts, respectively. All these monodic versions were then set into the same frequency range. Again, this modification did not change the ratings much: I.R.'s ratings were 3.2 and 8.8 for the sad and happy excerpts, respectively, and controls' ratings were 3.0 and 8.5.
} 
demanding in requiring precise and sustained monitoring of the input. In other words, emotional judgments may be easier to perform than non-emotional ones because they are continuously and consistently fed by redundant information.

To test the idea that I.R. exploits the redundancy that is present in the stimuli to achieve near-normal emotional judgments, she was presented again with the same task but this time the musical information was presented to her in segments of variable length. The segments always started at the beginning of the excerpt and increased systematically by $1 \mathrm{~s}$ from 0.5 to $7.5 \mathrm{~s}$. There were eight segments of different lengths for each beginning of the 32 different excerpts, resulting in a set of 256 trials. The segments were presented in a random order. After presentation of each segment, subjects were instructed to write down their happy-sad judgment on a 10-point scale. The session lasted about 2 h. I.R. was tested twice with the same set of trials, one year apart. That was done in order to increase the number of observations per response category.

The prediction was that if emotional judgments could be achieved by a deficient processing system because musical stimuli afford redundant information in this respect, then I.R. should exhibit clear evidence of a length effect. That is, the longer the segment, the more cues for determining its emotional category, and hence, the higher the confidence in the emotional judgments. Although normal controls were expected to exploit redundancy to some extent, I.R. should rely more heavily on this perceptual affordance than normals, given her impaired music processing system.

\subsubsection{Results and discussion}

I.R.'s pattern of performance did not differ between the two sessions and her data were collapsed in the following analyses. The mean ratings obtained by I.R. and her controls at each segment duration for the 'happy' and 'sad' selections are presented in Fig. 1. The averaged ratings obtained for each segment by I.R. and averaged across controls were submitted to two separate ANOVAs, both considering items as the random variable, Length (from 0.5 to $7.5 \mathrm{~s}$ ) as the within-items variable and response Category (happy vs. sad) as the between-items variable. The analyses revealed that both I.R.'s and controls' responses are sensitive to segment length; the interaction between Length and Category of the excerpt reached significance for I.R. $(F(7,210)=2.17, P<0.05)$ as well as for controls $(F(7,210)=6.94, P<$ 0.001); in fact, each control subject displayed this trend (see Fig. 1). As can be seen in Fig. 1, the length effect seems more pronounced for the 'happy' excerpts than for the 'sad' excerpts, particularly for I.R. Further analyses confirmed these observations: For I.R., the Length effect reaches significance for the happy excerpts only $(F(7,105)=5.59, P<0.001$; for the sad excerpts, the $F(7,105)=1.52$ is not significant (n.s.)). For controls, a length effect is obtained for both happy and sad excerpts (with $F(7,105)=5.65$ and $2.5, P<0.02$, respectively). These results are consistent with the slopes of the linear regression functions presented in Table 4. Contrary to our prediction, however, I.R.'s slopes are not steeper than those observed in controls. Her slopes fall within the $95 \%$ confidence interval of slopes seen in the normal controls for the happy excerpts and outside the interval for the sad excerpts. In the latter case, there is no slope in I.R.'s judgments. 
All subjects' judgments improve somewhat with increased durations, thereby supporting the notion that subjects use the redundant information contained in the excerpts to infer the emotional character of the piece. Further support to this notion is provided by the observation that subjects' ratings for the $7.5 \mathrm{~s}$ segments are not yet as extreme as those obtained for the full excerpts (mean duration: $15.8 \mathrm{~s}$ ) that were used in the previous experiments. However, all subjects, including I.R., are able to derive an appropriate emotional evaluation on the basis of the musical information that is contained in a $500 \mathrm{~ms}$ segment. At this brief duration, the subjects are able to

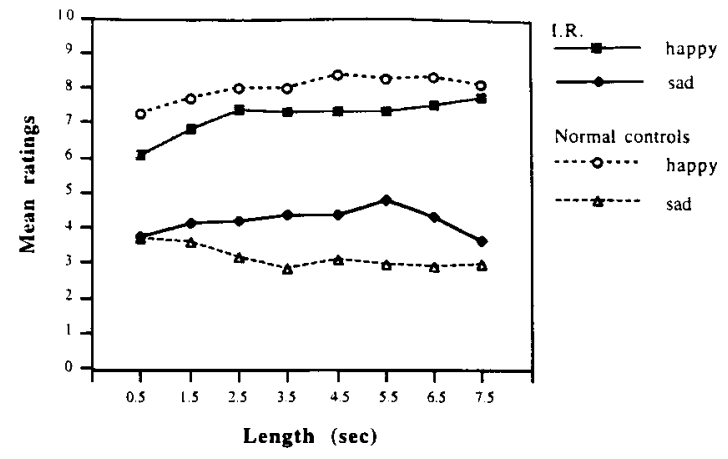

Control \#1

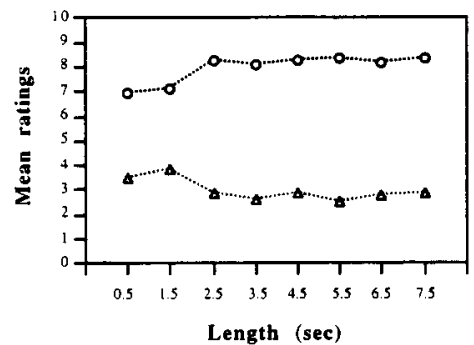

Control \#3

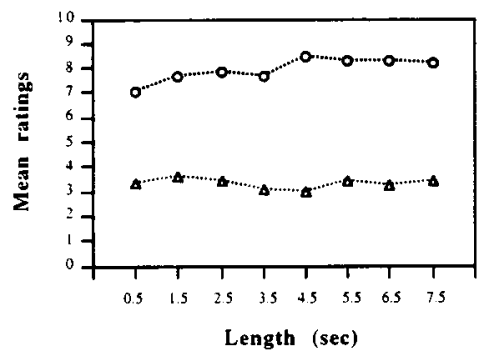

Control \#2

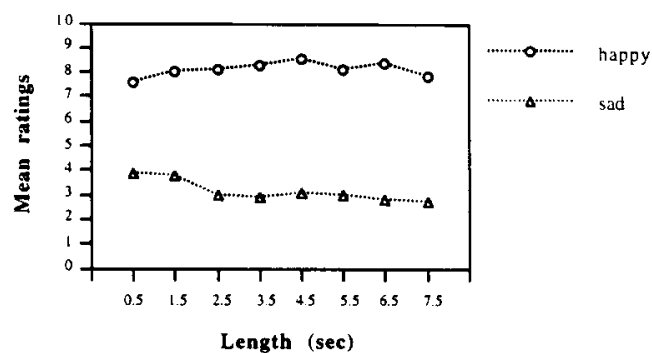

Control \#4

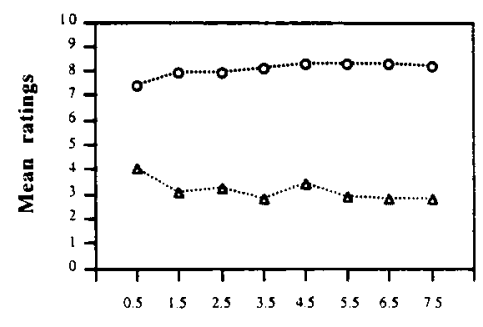

Length (sec)

Fig. 1. Mean ratings for happy and sad excerpts provided by I.R. and her controls as a function of stimulus length measured from onset, and presented in Expt. 3. The top figure represents I.R.'s data averaged across two sessions along with the averaged data of the controls. Below, the averaged data of each control subject are represented. 
Table 4

Linear regression analyses of the ratings as a function of the length of the musical segments

\begin{tabular}{lllcll}
\hline Subjects & Category & Intercept & Slope & $\begin{array}{l}\text { 95\% confidence } \\
\text { interval }\end{array}$ & $\begin{array}{l}\% \text { of } \\
\text { variance }\end{array}$ \\
\hline Controls & Happy & 7.5 & 0.13 & $0.05 / 0.20$ & 66 \\
\multirow{2}{*}{ I.R. } & Sad & 3.6 & -0.10 & $-0.17 /-0.04$ & 65 \\
& Happy & 6.5 & 0.18 & + & 70 \\
& Sad & 4.1 & 0.02 & - & 2 \\
\hline
\end{tabular}

The intercept, slope and $95 \%$ confidence interval for the slope (lower/upper limits) of the linear regression function (all given on a 10-point scale) as well as the percentage of variance it accounts for are presented separately for happy and sad selections. Note that for I.R.'s results, the confidence interval column indicates whether her slope falls within $(+)$ or outside $(-)$ the interval established for the normal controls in the corresponding category.

reliably distinguish 'happy' from 'sad' excerpts (with $t_{30}=4.500$ and 7.850 , both $P<0.001$ by bilateral tests, for I.R. and controls, respectively), although I.R.'s ratings appear slightly less extreme. Her scores are one standard deviation below normals for the 'happy' excerpts while they fall within the normal range for the 'sad' 0.5 s segments.

The fact that all subjects are able to discriminate 'happy' from 'sad' excerpts with as little as half a second of music is impressive. To better understand this result, we examined whether the $0.5 \mathrm{~s}$ segments contained clues related to the two major determinants of emotional judgments that were identified, namely, tempo and mode. For example, slow and fast tempi entail variations in density (i.e. in the number of sequential events, defined as one tone or chord, contained in the segment). The $0.5 \mathrm{~s}$ segments contained indeed, on average, 1.7 and 4.4. events in the 'sad' and 'happy' categories, respectively (the difference being significant, with $\left.t_{30}=2.090, P<0.05\right)$. Thus, subjects can, in principle, infer the pace of the whole excerpt by weighting the density of events contained in these very short segments. Mode is less straightforward. In theory, the mode could be inferred from very limited information as long as the segment incorporates a tonic triad or a third interval (major or minor). From this music-theoretical perspective, 14 of the 16 happy segments contained sufficient information to allow mode inference, while only 5 of the 16 sad segments did so. We checked this formal analysis with the judgments provided by four professional musicians. They were asked to rate on a 10point scale whether each of the $0.5 \mathrm{~s}$ segment was written in a minor or a major mode (with 1 meaning clearly minor and 10, clearly major). Musicians gave an averaged rating of 9.5 for the happy segments and of 5.7 for the sad ones, the difference being significant $\left(t_{30}=5.139, P<0.001\right)$. As expected from the theoretical analysis, the mode is easy to infer from the happy brief segments. For the sad brief segments, the mode is ambiguous since the average rating falls in the middle of the scale and variability is high (standard deviation is 2.86 and 0.85 , for the sad and happy segments, respectively). In summary, tempo and, to some extent, mode information can be used in the $0.5 \mathrm{~s}$ segments to determine the emotional tone. This strategy should be most effective for the happy than sad segments in which mode information 
is more salient. As can be seen in Fig. 2 and in Table 4, I.R.'s data square with this prediction.

The major conclusion to draw from this set of results is that emotional evaluation of a musical selection can be quickly established, since it can be made on the basis of very little information. The initial portion of the music seems to contain sufficiently distinctive information to allow accurate emotional evaluation of the whole excerpt. Moreover, and contrary to expectations, I.R. does not seem to proceed differently from normals; she does not appear to exploit redundancy to a greater extent than normals do. In other words, redundancy cannot fully explain the ease with which I.R. achieves emotional evaluation.

\subsubsection{Control conditions}

To assess whether the remarkable performance of the subjects in the $0.5 \mathrm{~s}$ window is a true reflection of emotional immediacy and is not due to some prior exposure effect with the musical excerpts, we ran two control conditions with university female subjects. In one control condition, ten naive students were presented with the same procedure as the one used with I.R. and her controls. We will refer to it as the random condition. The advantage of the random presentation format is that it minimizes decisional biases. Its disadvantage is that some of the shortest segments may beneficiate from prior exposures to longest segments of the same stimulus in previous trials. This is not the case in the standard procedure of the gating paradigm (Grosjean, 1980) in which the stimulus is presented progressively. In the standard procedure, to which we will refer as the successive condition, each excerpt is presented repeatedly by increasing its presentation time (duration from onset) at each successive pass. This successive condition was used with the other group of 10 naive

RANDOM

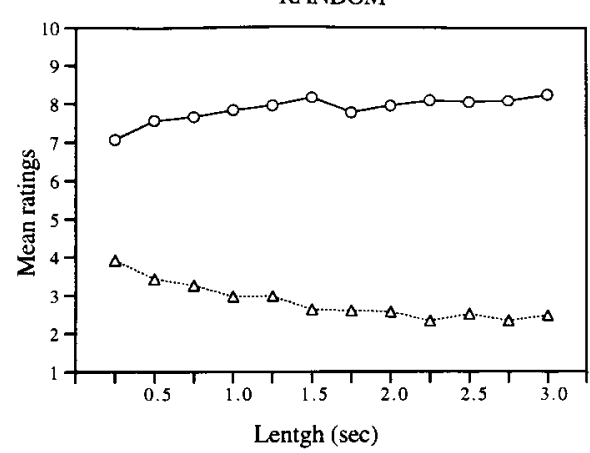

SUCCESSIVE

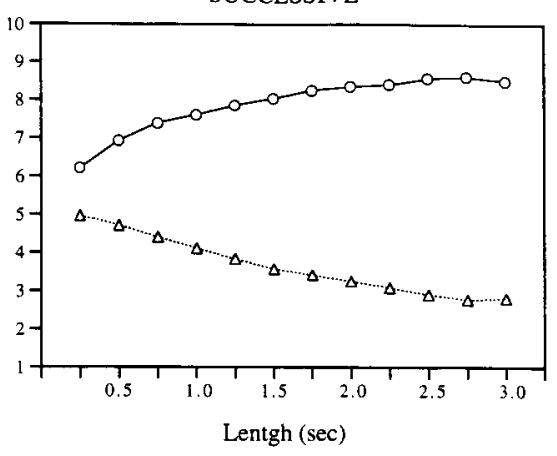

Students

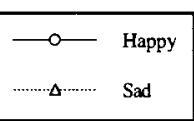

Fig. 2. Mean ratings for happy and sad excerpts provided by naive students as a function of stimulus length measured from onset, in the random and successive format of presentation. These conditions served as control data in Expt. 3. 
students. In both the random and the successive condition, the subject's task is to provide a confidence rating on the emotional tone after each segment.

In both conditions, two different orders of trial presentation were used. The stimuli were also presented in a smaller time scale than they were with I.R. and her controls. The incremental value was set to $250 \mathrm{~ms}$ (instead of $1 \mathrm{~s}$ ), so as to obtain a finer-grained measure. The segments always started at the beginning of the excerpt and increased systematically by $250 \mathrm{~ms}$, from 0.25 to $3.0 \mathrm{~s}$. There were 12 segments of different lengths for each of the 32 excerpts, resulting in a set of 384 trials.

As can be seen in Fig. 2, the subjects of the random condition gave very similar judgments to those obtained by I.R. and her matched controls. They even reliably distinguished the emotional tone of the $0.25 \mathrm{~s}$ segments, with $t_{9}=7.427$ and $t_{30}=$ 6.737, $P<0.001$, computed per subjects and items, respectively. In the successive condition, it is apparent that subjects were more conservative, showing evidence of a larger increase in confidence ratings as a function of segment length. These differences in confidence between the random and the successive format of presentation was expected since similar results have been reported for word recognition (Walley et al., 1995). What is more important in the present context is that subjects in the successive condition also display evidence of emotional discrimination of the $0.25 \mathrm{~s}$ segments; despite their conservatism, they reliably distinguished the emotional tone of the initial shortest segments, with $t_{9}=5.619$ and $t_{30}=5.122, P<0.001$, per subjects and items, respectively. This different treatment of the happy and sad brief segments cannot be due to prior exposure with the material since the excerpts had not been presented in previous trials. Therefore, the control data provide strong support to the notion that emotional evaluation along the happy-sad dimension is immediate.

\subsection{Experiment 4: discrimination judgments}

I.R.'s emotional treatment of music is impressive compared to her extensive loss of perceptual and memory abilities for music. In prior studies (Peretz et al., 1997; Patel et al., 1998; Peretz and Gagnon, 1998; see the discrimination tasks in Table 1), we showed repeatedly that I.R. failed to discriminate two successive musical sequences as same or different, whether the difference occurs on the pitch or on the temporal dimension. The major goal of the present experiment was to assess the extent to which I.R.'s discrimination difficulties generalize to the present material.

In all our previous work done with I.R., we used simple musical material. That is, the material was systematically monodic, short and rather stereotyped in structure. The present material is long, original and harmonically elaborated. It may be the case that I.R.'s deficient music processing system deals more effectively with such a rich musical material than with the impoverished one used in prior studies. Furthermore, in our previous investigations, the comparison melody incorporated only a small change (on one or two tones) with respect to the standard melody. Only a very small portion of the stimulus contained the critical information for discrimination. As suggested in Expt. 3, I.R., as any other brain-damaged patient, may need redundancy in order to process musical information more effectively. Therefore, the 
discrimination task was arranged so as to allow her to exploit redundancy for differentiation.

To this aim, I.R. was presented with a subset of the excerpts used in Expts. 1 to 3, arranged in a same-different classification task. When different, the successive excerpts were totally different (i.e. by every single event) while pertaining to the same genre and to the same emotional category. For example, a 'different' trial consisted of the Mozart's piano concerto \#27 followed by Mozart's piano concerto \#23 (both conveying a happy tone). In addition, we manipulated the duration of the inter-stimuli interval (ISI) so that the strength of the memory traces for the excerpts could be examined. The ISI varied from 4 to $20 \mathrm{~s}$ for each pair; the pairs and ISIs were presented in a random order. There were 36 trials for each ISI, half of which were 'different' trials, resulting in a set of 180 trials. The experiment lasted 2 hours.

\subsubsection{Results and discussion}

Two normal controls were tested in the same conditions. The task was so easy for them that they took the test while having a conversation with the experimenter during the retention interval. Normal controls, thus, performed at ceiling. This was not the case for I.R., who constantly complained about the difficulty of the task and who requested several pauses during the session. Her responses were considered as hits when she responded 'different' to a different trial and as false alarms when she responded 'different' to a trial made of identical excerpts. I.R.'s responses are represented in Fig. 3.

As can be seen in Fig. 3, I.R.'s performance is far from ceiling. Overall, she obtained 61 hits and made 20 false alarms (F.A.) (out of 90 trials in each category), hence performing below normal controls (who obtained 90 hits and 0 F.A.; $\left.\chi^{2}=16.69, P<0.001\right)$. To statistically assess whether I.R. shows sensitivity to time delay, her responses were submitted to an ANOVA considering Delay (4 vs. 8 vs. 12 vs. 16 vs. 20 s of ISI) and Response type (hits vs. false alarms) as withinitems variables. The analysis reveals no significant effect and no interaction. I.R.'s poor discrimination is probably not due to trace decay over time. I.R.'s performance is more compatible with a poor encoding of the excerpts in the first place.

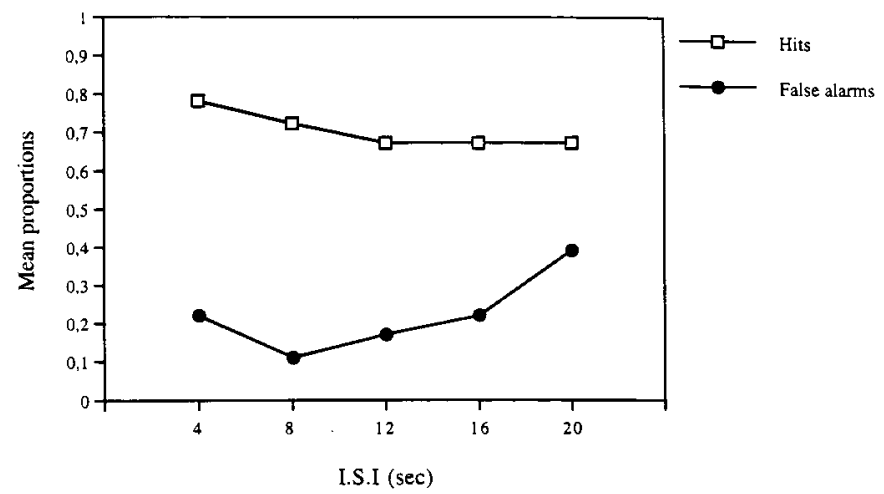

Fig. 3. Mean proportions of hits and false alarms made by I.R. in the same-different classification task used in Expt. 4. 
Finally, to explore the role of emotion on discrimination, I.R. was presented 6 months later with a similar discrimination task; this time, the successive excerpts, when different, also conveyed opposite emotional tone. For example, a 'different' trial consisted of the excerpt taken from the second movement of Mozart's piano concerto \#27 followed by the excerpt taken from the third movement of the same piece; the first excerpt corresponded to a 'happy' stimulus and the comparison excerpt to a 'sad' one. In this case, I.R. was $94 \%$ correct at an ISI of $20 \mathrm{~s}$ (with 18 hits and 2 false alarms). Yet, I.R. still complained that the task was very difficult. In fact, she was puzzled by her results because she thought she was only guessing. This result suggests that I.R. improves when her judgments are mediated by emotional evaluation (without awareness or intention).

\subsection{Experiment 5A: error detection task}

I.R.'s poor discrimination of the excerpts when arranged in a 'same-different' classification task is consistent with prior results obtained with her on other materials (Peretz et al., 1997; Peretz and Gagnon, 1998). This problem in discriminating successive musical stimuli cannot be entirely attributed to a memory deficit since increasing the retention interval did not decrease performance to an important degree. I.R.'s impairment seems to have a more perceptual origin, as we have suggested elsewhere (Peretz et al., 1997) and reported in Table 1 (see the scaleerror detection and tonal closure tests that are on-line perceptual tasks). If this is the case, then I.R. should also exhibit deficits in on-line perceptual tasks when dealing with the same complex musical excerpts used in the previous experiments. To test this prediction, we devised an error detection task. Errors were designed so as to be striking (and often hilarious) and plausible. This was done by modifying the pitch or the starting time of the notes contained in a single measure so as to mimic a pianist who, for a short moment, is completely 'off-the track', by either playing the wrong notes or playing off beat. The instruction was to monitor each excerpt and to decide whether or not the pianist was 'off-the-track or absent-minded' at some point during the performance.

\subsubsection{Material and procedure}

Twenty-one excerpts were selected so as to allow deviation of a single measure. To provide as much context as possible, the deviation never occurred on the initial and last measure of the excerpt. The changes affected all voices in ten excerpts and the leading voice (thus leaving the accompaniment unchanged) in the other 11 excerpts (see Fig. 4 for an illustration of a leading voice change). Two distinct types of deviations were applied to the same measure: either the pitch of each note was randomly set to a different pitch in the same frequency range or the time onset of each note was randomly changed in the same sequential position (the transformation was made by means of the random function of the Sequencer Plus Gold program). The resulting pitch changes were mostly out of key and the time changes were mostly off beat.

Each excerpt was presented in three formats: one intact (containing no change), 

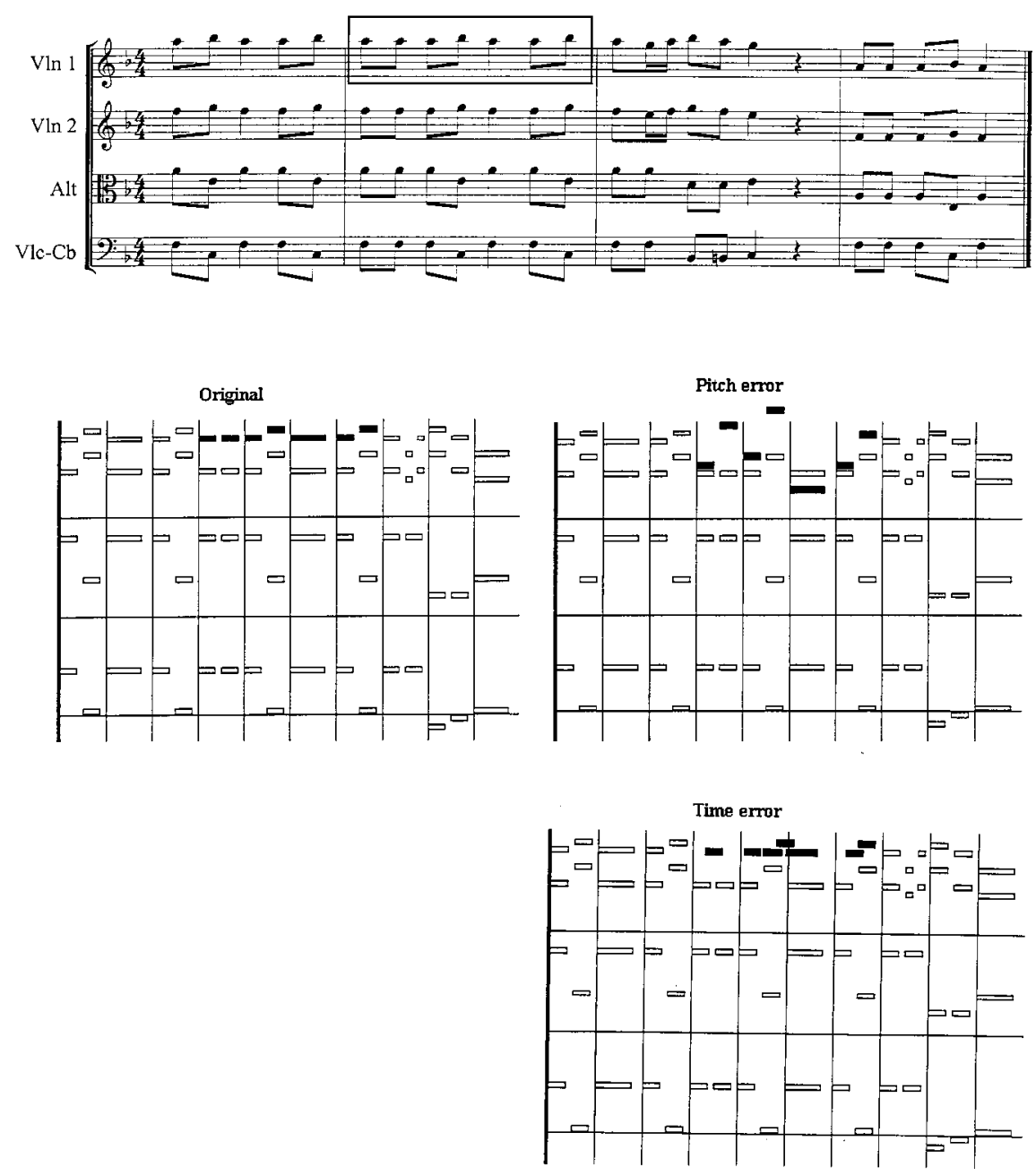

Fig. 4. Musical and analog representation of the excerpt taken from the 'Autumn' of Vivaldi to illustrate the nature and the extent of the deviations (in filled rectangles) applied to the melodic line of a whole measure used in Expt. 5A.

one with a pitch deviation, and one with a time deviation. In the latter two formats, the modified measure was the same (see Fig. 4). The intact format was presented twice so that half of the trials contained no error and the other half contained an error, which affected the pitch dimension or the time dimension equally often. There were 84 trials which were mixed in a random order. The subjects were requested to detect whether or not the pianist lost track of what he was playing at some point in the piece. There were two examples before the experimental ones. Subjects were not informed of the nature of the changes and no feedback was provided, except for the two examples. 
Table 5

The percentages of hits ('yes' to excerpts containing an error) and false alarms ('yes' to intact excerpts) for I.R. and her matched controls in the pitch and time error detection task used in Expt. 5A (random change) and 5B (shift change)

\begin{tabular}{|c|c|c|c|c|}
\hline & & I.R. & \multicolumn{2}{|c|}{ Matched controls } \\
\hline \multirow[t]{3}{*}{ Hits } & Time error & 33.3 & 73.8 & $* * *$ \\
\hline & Pitch error & 57.1 & 89.3 & $* *$ \\
\hline & Mean & 42.9 & 82.1 & \\
\hline False alarms & & 7.1 & 5.4 & $*$ \\
\hline \multicolumn{5}{|c|}{ B. Shift change } \\
\hline \multirow[t]{3}{*}{ Hits } & Time error & 30.0 & 71.4 & $* * *$ \\
\hline & Pitch error & 30.0 & 70.3 & $* *$ \\
\hline & Mean & 30.0 & 70.8 & \\
\hline False alarms & & 10.0 & 8.1 & $*$ \\
\hline
\end{tabular}

A: $* * * \chi^{2}=30.1, P<0.001 ; * * \chi^{2}=24.38, P<0.001 ; *$ not significant.

B: $* * * \chi^{2}=32.6, P<0.001 ; * * \chi^{2}=30.89, P<0.001 ; *$ not significant.

\subsubsection{Results and discussion}

The responses were scored as hits when the subjects correctly detected an error and as F.A. when they responded 'error' for an intact excerpt. The percentages of hits and F.A. are presented in Table 5 (the random change part). Both I.R. and control subjects were able to detect the deviations since they produced significantly more hits than F.A. ( $t_{20}=4.543$ and 18.065 , for I.R. and controls, respectively). However, I.R. made significantly fewer hits than controls $\left(\chi^{2}=30.81, P<0.001\right)$, therefore confirming her deficit in perceptual organization of music.

Hit rates were further analyzed to measure the potential effect of the nature of the deviations applied to the stimuli. It was found that control subjects detected better the pitch deviations than the time deviations (see Table 5; $t_{40}=2.219, P<0.05$ as computed per item). I.R. displayed the same trend; the advantage for the pitch over the time deviations was, however, not reliable $\left(t_{40}=1.240\right.$, n.s.). Finally, keeping the accompaniment unchanged helped I.R. and her controls to detect a deviation, particularly when the deviation affected the time dimension. However, the differences did not reach significance.

\subsection{Experiment 5B: error detection task}

In the previous task the errors were very striking, particularly in the pitch dimension. In this experiment, our goal was to equalize performance in the pith and time dimension and, above all, to provide further evidence that I.R. is impaired on such on-line monitoring tasks. To do so, we simply shifted either the pitch or the time onset of all tones of the leading voice comprised in a measure, instead of changing them randomly as in Expt. 5A. As these errors are more systematic (i.e., mimicking a pianist's right hand either spatially displaced or temporally desynchronized with 
respect to his left hand for a short moment), they were expected to be a little less salient than the random changes used previously.

\subsubsection{Material and procedure}

These deviations were applied to the same measure in the same 21 excerpts as in Expt. 5A. These changes affected only the leading voice. Two distinct types of changes were applied to the same measure: either the pitch of each note was shifted higher or lower by a semi-tone or the time onset of each note was set too early or too late by two-thirds of a quarter value (mean change: $320 \mathrm{~ms}$; range: 96-686). These transformations were made by means of the shift function of the Sequencer Plus Gold program. The resulting pitch errors were mostly out of key (for $60 \%$ of the modified tones, on average) and the time errors were systematically off beat. Thus, there were four modified versions for each intact excerpt: two versions with a pitch shift (one higher and one lower) and two versions with a time shift (one earlier and one delayed). These 84 modified excerpts were randomly mixed with 84 intact versions (corresponding to the 21 intact excerpts repeated four times). The instructions were the same as in Expt. 5A. The session lasted approximately $2 \mathrm{~h}^{4}$

\subsubsection{Results and discussion}

The responses were scored as in Expt. 5A and are presented in Table 5 (the shift change part). As can be seen, I.R. exhibits again a clear deficit in detecting both pitch and time violations. She succeeds to obtain more hits than false alarms, with $t_{20}=2.718, P<0.02$, although this success is by no means impressive. These results provide converging evidence that I.R. is impaired in the perceptual analysis of musical structure in a non-emotional context.

\subsection{Experiment 6: change monitoring task}

We showed that I.R. was deficient in discriminating the excerpts (Expt. 4) and in detecting obvious errors inserted in the excerpts (Expt. 5A,B). Both sets of results support the presence of severe deficits in processing music for other purpose than emotional evaluation. Moreover, the data obtained in Expt. 5A,B replicate previous results (e.g. Table 1) in showing that the processing deficits affect both the pitch and the time dimensions. The ensuing question is how could I.R. use structural characteristics such as mode and tempo when performing the 'happy-sad' judgments, while failing to build an accurate representation of the same excerpts in terms of their pitch and temporal information?

By most accounts, tempo evaluation requires a proper interpretation of beat (Dowling and Harwood, 1986; Drake and Botte, 1993). Similarly, mode perception requires encoding of pitch in musical scales (e.g. Dowling and Harwood, 1986). Accordingly, a deficiency in musical pitch and time organization should compromise mode and tempo evaluation. I.R.'s judgments do not seem to respect this

\footnotetext{
${ }^{4}$ Experiment 5B was run 1 year after completion of the present study. Anecdotally, during testing, I.R. inquired about having ever heard these musical excerpts before.
} 
principle. She exhibits normal sensitivity to mode and tempo (Expt. 2) while showing poor sensitivity to pitch and time regularities (Expt. 5A,B). However, the processing of these different musical properties was assessed with different tasks, one relying on emotional evaluation (Expt. 2) and the other on error detection (Expt. 5). Thus, task-related factors may explain the observed dissociation in I.R.

In order to remedy this situation, I.R. and her controls were presented with a novel error detection task in which the error consisted of a change in mode or in tempo of a single measure of the musical excerpt. These changes were applied to the same measures that bore the errors in Expt. 5, and obeyed the same principles as those applied to the whole excerpts in Expt. 2. Since mode and tempo changes are musically legal in the sense that they do not violate the rules of the Western tonal musical system, it is more appropriate to refer to this task as a change monitoring task rather than an error detection task.

\subsubsection{Material and procedure}

Thirty excerpts (including the 21 excerpts used in Expt. 5) were selected. The changed measures were the same as the ones modified in Expt. 5. Three types of deviation were applied to these measures in each excerpt: either the mode was changed (from major to minor and vice versa), the tempo was changed (set to the value of 84 M.M.), or both mode and tempo changes were combined. All events in the modified measure were affected by the tempo deviation. In contrast, an average of $48 \%$ of these same events were modified by mode inversion. Thus, detection of a mode change was expected to be more difficult than that of a tempo change. Each type of change was tested in a different condition. Each condition consisted of 60 trials, half of which corresponded to intact trials.

Presentation followed an increasing order of difficulty, the mode + tempo condition being first, the tempo and the mode condition being second and last, respectively. Subjects were not informed about the nature of the changes. Instructions, which were similar to those used in Expt. 5, required the subject to detect whether or not the pianist was deviating from what he was expected to play. Subjects were simply warned that the task was more subtle than the previous one. Two examples were introduced at the beginning of the test to illustrate the difficulty of the task.

Table 6

The percentages of hits ('yes' to excerpts containing a change) and false alarms (F.A.) ('yes' to intact excerpts) for I.R. and her matched controls in the tempo and mode change monitoring task used in Expt. 6

\begin{tabular}{|c|c|c|c|c|}
\hline & \multicolumn{2}{|l|}{ I.R. } & \multicolumn{2}{|c|}{ Matched controls } \\
\hline & Hits & F.A. & Hits & F.A. \\
\hline Tempo + mode change & 70.0 & 10.0 & 71.7 & $14.3^{\mathrm{a}}$ \\
\hline Tempo change & 50.0 & 6.7 & 73.3 & $11.0^{\mathrm{b}}$ \\
\hline Mode change & 46.7 & 6.7 & 50.8 & $11.0^{\mathrm{c}}$ \\
\hline Mean & 55.6 & 7.8 & 65.3 & 11.9 \\
\hline
\end{tabular}

${ }^{\mathrm{a}, \mathrm{c}}$ Not significant.

${ }^{\mathrm{b}} \chi^{2}=6.5, P<0.02$. 


\subsubsection{Results and discussion}

The responses were scored into hits and F.A., as in Expt. 5. The results obtained by I.R. and her controls are displayed in Table 6 . As can be seen, all subjects are able to detect changes, by obtaining more hits than F.A. (all $P<0.01$ by binomial tests). These responses were analyzed by separate ANOVAs for I.R. and controls, respectively. Response Category (hits vs. F.A.) and Condition (tempo + mode vs. tempo vs. mode) were considered as within-items factors. For I.R., the analysis reveals a main effect of Condition $(F(2,58)=3.2, P<0.05)$ and no interaction between Category of response and Condition $(F<1)$. As shown in Table 6, I.R. performs best when tempo and mode changes are combined. For controls, the analysis yields an interaction between response Category and Condition, with $F(2,58)=6.01$, $P<0.005$. This interaction reveals that controls' F.A. rates do not differ across conditions $(F<1)$ while their hit rates differ $(F(2,58)=7.96, P<0.001)$. As shown in Table 6, normal controls performed best when tempo deviations are inserted either alone or in combination with mode changes.

Finally, I.R.'s results were compared to the controls' results by way of $\chi^{2}$ tests (see Table 6). Her performance was found to be below that of controls in the tempo condition only. ${ }^{5}$

The responses were examined more closely to assess the role of various factors that may have influenced the detection of the changes. With respect to tempo, the direction of change (i.e. the change producing a local decrease or increase in tempo, depending on the original tempo) was found to have an effect. Controls obtain a hit rate of 0.83 and 0.66 on decelerated and accelerated tempi, respectively. I.R. exhibits a similar trend, with 0.66 vs. 0.39 hit rates for the decelerating and accelerating changes. However, the differences do not reach significance $\left(t_{28}=1.385\right.$ and 1.496 , for controls and I.R., respectively, both n.s.). These trends are consistent with the literature showing that a decrease in tempo is more effectively detected than an increase in tempo (see Duke, 1989 for a review). This effect is attributed to the general propensity of musicians to deviate from fixed tempi by increasing, rather than decreasing, the speed of the beat. In the mode condition, we did not find context effects; that is, changes to a minor mode in a major mode context were not easier to detect than minor mode local changes in major contexts. We found, however, that the more the modified events contained in the changed measure, as dictated by mode transcription, the better the performance. In fact, proportion of changes varied from $13 \%$ ( 1 event out of 8 was modified) to $100 \%$ (all events were changed). To assess the influence of this variable, trials were split in two parts, with one half containing few changes and the other, many changes. Control subjects obtain hit rates of 0.30 and 0.72 for the few and many changes, respectively $\left(t_{28}=3.411, P<0.002\right)$. Again, I.R.'s results exhibit a less pronounced but similar trend, with 0.40 and 0.53 hit rates for the few and many changes; the difference is, however, far from significant $\left(t_{28}=0.713\right.$, n.s. $)$.

I.R.'s overall pattern of results is consistent with the monitoring of both tempo and mode information. As can be seen in Table 6, I.R. performed best when mode

${ }^{5}$ We replicated I.R.'s results 5 months later. 
and tempo deviations were combined. This pattern is analogous to the one obtained for emotional judgments in Expt. 2 (see Table 3) where changes in mode and tempo had the largest effect on performance when combined than isolated. Normal controls did not follow this pattern here (see Table 6). In fact, each control obtained slightly more hits in the tempo condition than in the mode + tempo condition. Normal controls seem to have used a slightly different strategy, focusing perhaps more on tempo than mode, and /or consulting their memory representations of the music to help them conform to the demands of the task. Memory consultation is likely since subjects were repeatedly tested with the same material over two years. They probably have stored accurate memory representations of the stimuli over the course of testing. In contrast, I.R. most probably could not resort to such memory representations because these are degraded; her performance is thus mostly driven by the musical input.

The major conclusion to be drawn from this last experiment is that I.R. appears to be using the same structural cues - mode and tempo - in emotional and non-emotional tasks. This is not a trivial finding, as we will argue in the general discussion.

\section{General discussion}

The present study grew out of the observation of a remarkable sparing of emotional responses to music in the context of severe deficits in processing music as a result of brain damage. To understand the origin of this neuropsychological dissociation, a set of six experiments was designed to explore the perceptual basis of emotional judgments in music. In doing so, a number of findings were obtained. Some concern the functioning of a normal brain, since several experimental situations in the present study have never been investigated before. These results will be summarized first. The other findings, which relate to the effects of brain damage on emotional perception, will be discussed thereafter with respect to the possibility that emotional analysis of music is mediated by an isolable neural system.

As mentioned in the introduction, emotional processing of music is a largely neglected area of investigation in current experimental psychology (see Juslin, 1997 and Krumhansl, 1997 for reviews), including neuropsychology. This is a curious situation since music is essentially conceived as an emotional medium acting as a social cohesive force (Sloboda, 1985; Dowling and Harwood, 1986). Under this view, the major reason for music existence (or its survival) in human societies is likely to be related to its emotional roots. Accordingly, and contrary to the belief that emotional responses are highly variable from one individual to another, emotional responses to the music of one's culture are expected to be relatively homogeneous across individuals, being shared by all members of that culture. The results obtained in the present study are largely consistent with this prediction.

Emotional judgments are found to be remarkably consistent and reliable within and across subjects. That is, we found that every subject, who may represent the general population by her being a non-university and musically untutored indivi- 
dual, is capable of discriminating between the happy and the sad tone conveyed by music. These emotional classifications are not artificially induced by some manipulation of the material since the music was not purposely composed for the experiments. The musical stimuli were taken from the work of various established composers, spanning several time periods. The emotional classifications were, however, reduced to two basic categories, the happy and sad ones, which obviously do not cover the whole range of emotional responses to music. For example, there appears to be another basic category for fearful music (e.g. Krumhansl, 1997). Since fear is rarely conveyed by art music and is sometimes confused with anger (e.g. Terwogt and Van Grinsven, 1991), it was not studied. Rather, we opted for a simple and salient dichotomy in this initial exploration, the idea being to establish guidelines for future research with further emotional categories. This strategy enables us to conclude that, as far as the happy-sad categories are concerned, subjects recognize the distinction without hesitation, presumably by drawing on general and shared knowledge about these musical emotions.

Although subjects judged the excerpts as pertaining to the happy-sad categories seemingly with little effort, the way they accomplished these judgments appears extraordinarily more complex. We showed that the judgments relied on the computation of several and relatively elaborate musical properties. By manipulating the mode in which the excerpt was originally written and the tempo in which it was supposed to be rendered, we were able to show that both mode and tempo largely determined emotional classification along the happy-sad dimension. Change of mode (from major to minor, and vice-versa) and change of tempo (to a unique neutral value) both impaired emotional distinctiveness of the excerpts, particularly when both mode and tempo changes were applied jointly to the excerpts (Expt. 2). In contrast, human expressiveness and instrumentation were found to have negligible effects (Expt. 1). That is, whether the musical excerpt was interpreted by humans or generated without nuances by a computer did not influence the ratings to a significant degree. Similarly, whether the excerpt was presented in its original instrumentation or transcribed for a unique instrument (the piano) did not have a significant impact on emotional ratings either. Thus, emotional judgments appeared here to depend on the structure of the music itself and little on the manner in which it was expressed.

The proposal that mode and tempo are major determinants of emotional classification along the happy-sad dimension is not new, although it has not received much empirical support. The major piece of evidence dates back to Hevner's $(1935,1937)$ classical work. Hevner (1935) presented ten excerpts from the classical repertoire and asked subjects to select emotional adjectives that best described the emotional tone of the piece. Each excerpt was presented once in a major mode and once in a minor mode. Subjects were found to select more often the happy label when the piece was written in a major mode, and the sad label for the minor pieces. This distinction was found to be independent of musical training. In a subsequent study, Hevner (1937) presented eight excerpts, different from the ones used earlier, each being played at a slow (between 63 and 80 M.M.) and at a fast (from 104 to 112 M.M.) tempo. Subjects again assigned more often the happy label to the fast pieces and the serene (rather than sad) label to the slow pieces. Hevner concluded that from 
all the variables that she previously investigated, tempo is 'of greatest importance in carrying expressiveness in music. [....] Modality [mode] is perhaps second in importance' (p. 625). More recently, Scherer and Oshinsky (1977) reached similar conclusions. However, in neither study, the relative contribution of mode and tempo was assessed for the same selections, nor did Hevner assess the same subjects with both manipulations. In keeping the material and subjects constant, we were able to show that mode and tempo both contribute to the happy-sad distinction.

In general, more work has been devoted to mode than tempo as a structural determinant of the happy-sad distinction. Interestingly, the three studies (Kastner and Crowder, 1990; Gerardi and Gerken, 1995; Gregory et al., 1996) which have explored the influence of mode on emotional judgments converge on the same conclusion. In each study, a rather strong connection between the major-minor mode and the happy-sad distinction is obtained. Moreover, sensitivity to mode via emotional judgments appears precociously, around the age of 3 years (Kastner and Crowder, 1990). However, the other two studies (Gerardi and Gerken, 1995; Gregory et al., 1996) fail to replicate this precocious performance, but suggest instead, that the systematic relation between mode and emotion emerges later, at the age of 8 years. Nevertheless, it is worth emphasizing that mode, a rather elaborate property of the Western musical pitch system, is used with consistency across studies and subject populations in emotional judgments. This observation gives further support to the notion that emotional responses are rooted into common knowledge of music as an emotional language.

The role of human expression and instrumentation in conveying emotional tone has, to the best of our knowledge, never been assessed. In fact, their contribution to musical emotions is taken for granted. For example, Gabrielson and Juslin (1996) have studied in some detail how musicians convey different emotions by slightly and characteristically deviating from what is written in the score. The role of the interpreter as opposed to the contribution of the musical structure (i.e. the score) has not been assessed. The same applies to instrumentation. In the present study, we failed to find any significant influence of both human expression and instrumentation factors. However, it would be absurd to conclude, on the basis of these negative results, that interpretation and instrumentation do not play any role in emotional communication. Depending on the musical selections and on the ambiguity (or subtlety) of the structural content with regard to emotions, human interpretation may well play a decisive role. It is our contention, however, that structural content of most (popular) music will predominantly and unambiguously determine emotional responses.

One surprising finding in the present study concerns the rapidity with which subjects perform the happy-sad distinction. Subjects were found to be able to distinguish happy from sad music with as little as a half-second from the beginning of the music (Expt. 3). These initial portions contain very few musical events (from 1 to 4 ) but these events are distinctive ones. Sad initial segments are often limited to a single long-lasting event; happy initial segments contain several short events that typically index the mode of the piece. Apparently, subjects are able to pick up this information, as if the emotional character of the whole excerpt is determined by its 
very first sound(s). To provide an order of comparison, subjects need on average $2 \mathrm{~s}$ from the beginning of a highly familiar tune, such as that of 'happy birthday', to be able to identify it (Aronoff, 1997). Thus, recognition of emotion appears to be more immediate than recognition of identity, although both activities appear to be done without effort and without conscious reflection by the large majority of listeners.

Altogether, the results show that emotional classification of music is performed reliably and effectively by adults with moderate formal education and little musical training. These emotional judgments are determined by the structure of the music presented and are relatively immediate. Such a conclusion is rather uncommon in the study of non-musicians (Smith, 1997). That is, non-musicians' demonstrated abilities in the laboratory often fail to match in complexity and efficiency the ones presumed to be at work in natural settings. The present success with non-musicians, although tested in a laboratory with open-ended instructions, is, in all likelihood, related to the nature of the task required. Asking subjects whether the music evokes some emotional experience, or whether they like or dislike it (as we have done in Peretz et al., 1998), makes sense to non-musicians because this is one of the primary reason why they listen to music. Because emotional judgments are closer to daily experience with music, they may be more appropriate to reveal the content and the organization of the listeners' implicit knowledge of music structure than most nonemotional judgments typically required in the laboratory. Accordingly, emotional judgments may serve as a convenient tool for studying indirectly the content and organization of the music perceptual system of the majority of listeners.

However, emotional responses may not tap the perceptual system in the same manner as non-emotional judgments do. Zajonc (1980, 1984), for example, postulates separate affective and cognitive processing pathways. He proposes that affective processing of stimuli is a separate, quicker, and earlier-developing system than the cognitive system, both in individuals and in phylogeny. If this claim is correct, then the results obtained with emotional judgments would not reveal the functioning of the perceptual processing system. Rather, emotional judgments would reveal the functioning of a distinct, affective system. The current evidence is compatible with the notion of separate pathways. As mentioned above, emotional evaluation appears quick, precocious and homogeneously distributed across subjects. In contrast, nonemotional judgments (to which we will refer as to cognitive judgments), such as identity recognition, closure judgments, discrimination judgments, etc., appear somewhat later in development, are sensitive to musical expertise, and are probably slower. These differences are, however, only suggestive. More compelling evidence is generally provided by neuropsychological dissociations.

To the extent that emotional and cognitive judgments are governed by two partially independent systems, and that these systems are implemented in separable neural structures, brain damage may selectively disrupt one system and leave the other one intact. Such a logic underlies most reports of neuropsychological dissociations. If a patient can no longer recognize the musical input but is able to recognize its emotional tone, then the dissociation pattern argues for the processing autonomy of the recognition and emotion systems. The inference is even stronger if a different patient is found to display the reverse dissociation; that is, a loss of emotional 
appraisal in the presence of intact recognition. The two patients or dissociation patterns constitute instances of a double dissociation, which in turn provides strong support for the independent pathway proposal. Alternatively, if a single system underlies the emotional and non-emotional evaluation of music, any damage to this system should affect both types of evaluation along similar principles.

There is currently abundant evidence favoring the affective and cognitive separation view in other domains than music. In the face processing domain, brain damage can produce selective deficits in the recognition of identities while leaving intact the recognition of expressions. Inversely, selective deficits affecting the recognition of emotion from facial expressions without disturbing recognition of their identity have been reported (e.g. Etcoff, 1984; Parry et al., 1991; Young et al., 1993). These observations entail that certain aspects of face identity and facial expression are analyzed separately in the human brain. Analogous suggestions have been recently put forward for human voices (Imaizumi et al., 1997). In what follows, we will examine the extent to which this dissociation can be applied to music, in the light of the results collected with our amusic patient - I.R.

At first sight, I.R.'s results seem to conform to the separate pathways view. In a previous study (Peretz and Gagnon, 1998), I.R. was found to be impaired in the recognition of familiar popular melodies (e.g. the tune of 'happy birthday') while being able to derive their emotional tone. In the present study, I.R. showed an impairment in the processing of musical excerpts when these were arranged in a very easy same-different classification task (Expt. 4) and when the excerpts contained an obvious error (Expt. 5A,B). In contrast, for the same excerpts, I.R. provided normal emotional judgments (Expts. 1-3). These judgments relied on both mode and tempo cues as did those provided by neurologically-intact subjects (Expt. 2). Thus, I.R.'s pattern of results is consistent with current neuropsychological dissociations showing intact processing of emotional expression and impaired cognitive abilities. The results further suggest that perceptual analysis of the musical input is intact for emotional purpose and defective for non-emotional ones.

There is a problem, however, with this suggestion. I.R. is able to detect changes of both mode and tempo when locally applied to a measure of the musical excerpts (Expt. 6). These results are problematic for two reasons. First, I.R.'s results on the mode and tempo change monitoring task indicate that she can process mode and tempo in a nonaffective or cognitive context. Such a finding is difficult to reconcile with the hypothesis that I.R. exhibits a dissociation between spared affective and impaired cognitive processing. Secondly, I.R.'s pattern of results in the cognitive tasks is paradoxical. I.R. exhibits near-normal performance when a mode and a tempo change has to be detected (Expt. 6) while she displays poor performance when obvious violations of the musical structure, in pitch and time, are inserted in the same excerpts (Expt. 5A,B). This dissociation is difficult to reconcile with most accounts that consider tonal encoding of pitch and beat perception as intrinsic to the process of mode and tempo perception, respectively (e.g. Dowling and Harwood, 1986).

There are two different directions one may take in interpreting the observation that, despite deficits in pitch and time processing, I.R. is able to abstract mode and tempo, whether the task refers to emotional evaluation or not. One direction would 
be to argue that mode and tempo are computed independently from tonal encoding of pitch and beat perception, respectively. The other direction would be to argue that mode and tempo are essential, and thus mainly computed, for affective purpose. We will examine these alternative interpretations in turn.

In principle at least, tempo and mode can influence perceptual judgments without requiring music-specific knowledge. For example, tempo changes can be noticed on the basis of general-purpose mechanisms that do not require perception of regularity, or beat perception. One could compute relative durations of events or of intervals the way evaluation of the rate of speaking is done. By this view, I.R. would be able to notice changes in the rate of auditory events while failing to perceive regularity, and hence deviations from regularity, in music. A similar account can also be provided for mode perception. The harmonics of the notes of the major and minor tonic triads, which provide the strongest cues for mode, differ in consonance an/or in general familiarity. That is, the spectral components of the tones of the major scales match better and are more typical of the periodic sounds used in the environment. Such an acoustic account of the difference between the major and minor scales goes back to Rameau and Helmholtz (see Kastner and Crowder, 1990 for the development of similar ideas as applied to normal development, and Crowder, 1984 for a review). By this account, I.R. would be able to distinguish major from minor modes on a purely acoustical basis while failing to map pitch intervals onto musical scales. This explanation is not entirely satisfactory. For example, in the present study, the pitch deviations inserted in the error detection tasks (Expt. 5A,B) created dissonance. Such dissonance is noticeable on a similar acoustical basis, probably more so than is the minor mode. If I.R. were relying on acoustical features to reach normal levels of performance in discriminating major from minor modes, she should have performed similarly in detecting pitch errors. She did not. Therefore, a general-purpose account of I.R.'s way of processing music does not accommodate well her dissociation between intact mode perception and impaired tonal pitch perception.

The alternative direction one may take to interpret I.R.'s counterintuitive results is to consider her performance on mode and tempo as arising from the operations of a (spared) emotionally-relevant processing subsystem. There are several lines of argument that make this interpretation plausible. First, mode and tempo are two musical properties whose major functions may well be related to emotional appraisal. Tempo, for instance, is orthogonal to the process of music recognition; one can easily recognize a familiar melody despite important changes in speed. Second, I.R. may have used this affective pathway as a compensatory strategy; she may have learned through extensive testing of her emotional responses over the last two years that the emotional judgments correspond to the only musical area where she demonstrates spared abilities. When confronted with a novel musical task that lends itself to emotional evaluation, I.R. may have learned to draw back on these spared judgments even when these are not required by task demands. The use of such a compensatory strategy does not even need to be voluntary. Indeed, it has been suggested in Expt. 4 that I.R. relies without awareness on emotional distinctiveness in a 'same-different' discrimination task. Thirdly, we have anecdotal support for the view that emotions can mediate cognitive performance without awareness from 
another amusic patient whom we studied in detail (C.N. in Peretz et al., 1994; Peretz, 1996). When listening to the famous adagio of Albinoni taken from the patient's own record collection, C.N. first said that she had never heard that piece before. Suddenly, she said: 'it makes me feel sad...the feeling makes me think of the Albinoni's adagio' (Kolinsky, personal communication). C.N., who was totally unable to recognize melodies that were highly familiar to her before her brain accident, was able to do so via her emotional responses. We admit, however, that the use of emotion as a compensatory strategy is somewhat ad hoc in the present state of knowledge. To determine which of the two competing interpretations is the most fruitful will depend on future studies.

We can safely conclude from the present study that extensive brain damage can spare emotional appreciation of music. Emotional responses to music appear to recruit brain circuitries that are particularly resistant to damage. Such resistance should not, however, be equated with the functioning of a primitive sub-cortical system. Emotional appreciation of music requires, in all likelihood, the use of sophisticated knowledge of musical structure. Nevertheless, recourse to this knowledge appears to be immediate, and available to the layman without conscious reflection and with little effort. Therefore, emotional appreciation of music is best conceived as the product of a specialized cortical arrangement that is shared by most members of a given musical culture.

\section{Acknowledgements}

We are grateful to I.R. for her active and continuing cooperation. Excerpts of the present work were presented in the series 'Music and the Mind' and broadcast on I.T.V. (U.K.) in May 1996. We are grateful to Julie Ayotte for testing control students. We particularly wish to acknowledge the insightful and constructive comments made by Carol Krumhansl, Emannuel Bigand, Carolyn Drake and an anonymous reviewer on a previous draft of this paper. The research was supported by a grant from the Natural Science and Engineering Research Council of Canada to the first author and by a fellowship from the Fonds de la Recherche en Santé du Québec to the second author.

\section{References}

Aronoff, N., 1997. An Application of the Cohort Model to Melody Recognition. Unpublished Master's thesis, Université de Montréal.

Crowder, R.G., 1984. Perception of the major/minor distinction: I. Historical and theoretical foundations. Psychomusicology 4 (1-2), 3-12.

Dowling, W., Harwood, D., 1986. Music Cognition. Academic Press, New York.

Drake, C., Botte, M.C., 1993. Tempo sensitivity in auditory sequences: Evidence for a multiple-look model. Perception and Psychophysics 54, 277-286.

Duke, R., 1989. Musicians' perception of beat in monotonic stimuli. Journal of Research in Music Education 37, 61-71.

Etcoff, N., 1984. Selective attention to facial identity and facial emotion. Neuropsychologia 22, 281-295. 
Gabrielson, A., Juslin, P., 1996. Emotional expression in music performance: Between the performer's intention and the listener's experience. Psychology of Music 24, 68-91.

Gerardi, G.M., Gerken, L., 1995. The development of affective response to modality and melodic contour. Music Perception 12 (3), 279-290.

Gregory, A., Worral, L., Sarge, A., 1996. The development of emotional responses to music in young children. Motivation and Emotion 20, 341-349.

Grosjean, F., 1980. Spoken word recognition processes and the gating paradigm. Perception and Psychophysics 28, 267-283.

Hevner, K., 1935. The affective character of the major and minor modes in music. American Journal of Psychology 47, 103-118.

Hevner, K., 1937. The affective value of pitch and tempo in music. American Journal of Psychology 49, 621-630.

Imaizumi, S., Mori, K., Kiritani, S., Kawashima, R., Sugiura, M., Fukuda, H., Itoh, K., Kato, T., Nakamura, A., Hatano, K., Kojima, S., Nakamura, K., 1997. Vocal identification of speaker and emotion activates different brain regions. NeuroReport 8, 2809-2812.

Juslin, P., 1997. Emotional communication in music performance: A functionalist perspective and some data. Music Perception 14, 383-418.

Kastner, M.P., Crowder, R.G., 1990. Perception of the major/minor distinction: IV. Emotional connotations in young children. Music Perception 8, 189-202.

Krumhansl, C., 1997. An exploratory study of musical emotions and psychophysiology. Canadian Journal of Experimental Psychology 51, 336-353.

Liégeois-Chauvel, C., Peretz, I., Babaï, M., Laguitton, V., Chauvel, P., 1998. Contribution of different cortical areas in the temporal lobes to music processing. Brain, in press.

Parry, F., Young, A., Saul, J.S., Moss, A., 1991. Dissociable face processing impairments after brain injury. Journal of Clinical and Experimental Neuropsychology 13, 545-558.

Patel, A., Peretz, I., Tramo, M., Labrecque, R., 1998. Processing prosodic and musical patterns: A neuropsychological investigation. Brain and Language 61, 123-144.

Peretz, I., 1990. Processing of local and global musical information in unilateral brain-damaged patients. Brain 113, 1185-1205.

Peretz, I., 1996. Can we lose memories for music? The case of music agnosia in a nonmusician. Journal of Cognitive Neurosciences 8, 481-496.

Peretz, I., Gagnon, L., 1998. Dissociation between recognition and emotional judgments for melodies. Neurocase, in press.

Peretz, I., Kolinsky, R., Tramo, M., Labrecque, R., Hublet, C., Demeurisse, G., Belleville, S., 1994. Functional dissociations following bilateral lesions of auditory cortex. Brain 117, 1283-1302.

Peretz, I., Belleville, S., Fontaine, F., 1997. Dissociations entre musique et langage après atteinte cérébrale: un nouveau cas d'amusie sans aphasie. Canadian Journal of Experimental Psychology $51,354-368$.

Peretz, I., Gaudreau, D., Bonnel, A.-M., 1998. Exposure effects on music preference and recognition. Memory and Cognition, in press.

Scherer, K.R., Oshinsky, J.S., 1977. Cue utilization in emotion attribution from auditory stimuli. Motivation and Emotion 1, 331-346.

Sloboda, J., 1985. The Musical Mind: The Cognitive Psychology of Music. Oxford University Press, London.

Smith, J.D., 1997. The place of musical novices in music science. Music Perception 14, 227-262.

Terwogt, M.M., Van Grinsven, F., 1991. Musical expression of mood states. Psychology of Music 19, 99109.

Walley, A., Michela, V., Wood, D., 1995. The gating paradigm: Effects of presentation format on spoken word recognition by children and adults. Perception and Psychophysics 57, 343-351.

Young, A., Newcombe, F., de Haan, E., Small, M., Hay, D., 1993. Face perception after brain injury. Selective impairments affecting identity and expression. Brain 116, 941-959.

Zajonc, R.B., 1980. Feeling and thinking: preferences need no inferences. American Psychologist 35, 151-175.

Zajonc, R.B., 1984. On the primacy of affect. American Psychologist 39, 117-123. 


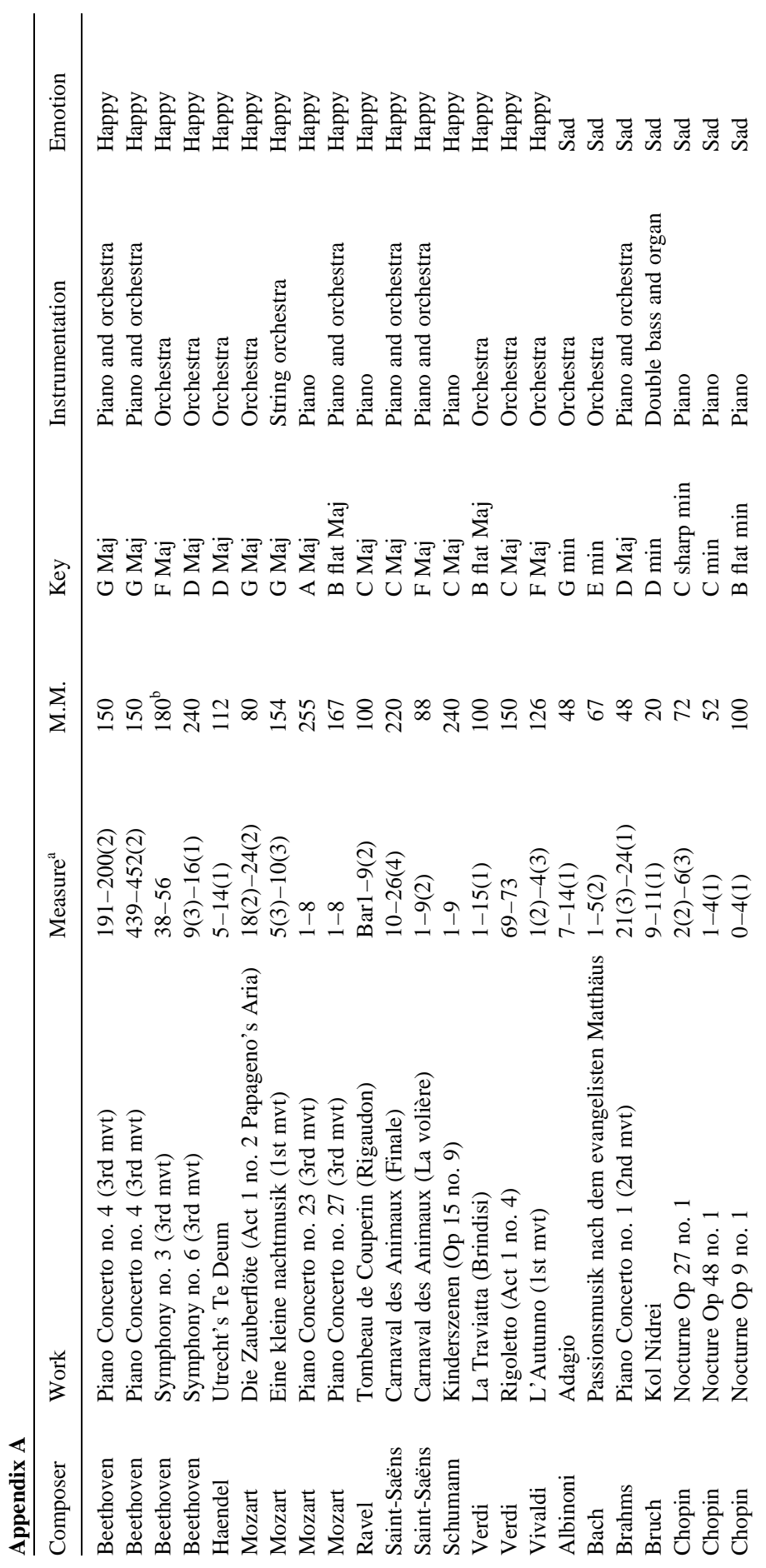




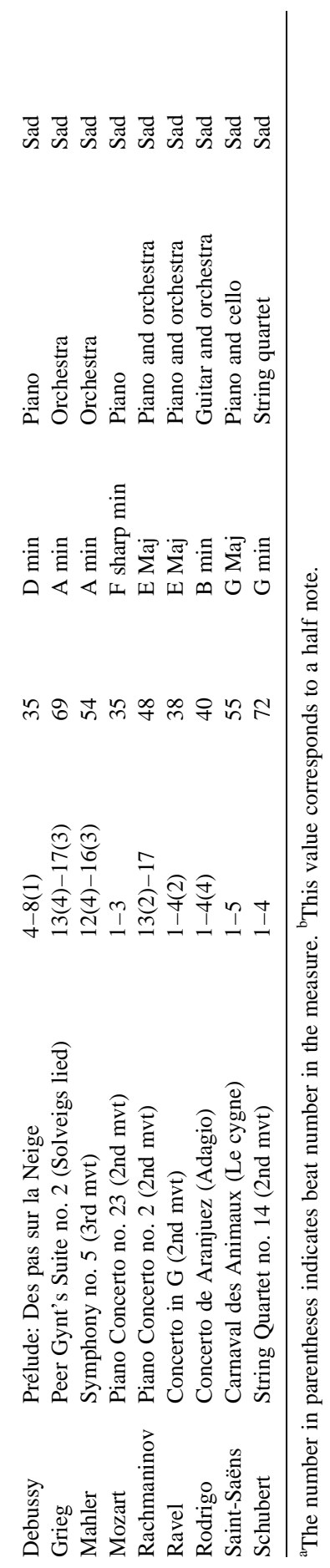

